\title{
Emergence and Evolution of Olfactory and Gustatory Symptoms in Patients with COVID-19 in the Outpatient Setting
}

\author{
Mahboobeh Karimi-Galougahi ${ }^{1}$ Ali Safavi Naini ${ }^{1} \cdot$ Jahangir Ghorbani $^{1}$. \\ Nasim Raad ${ }^{1} \cdot$ Negar Raygani $^{1}$
}

Received: 31 August 2020/Accepted: 21 September 2020/Published online: 28 September 2020

(C) Association of Otolaryngologists of India 2020

\begin{abstract}
The present study aimed at evaluating the prevalence of general and sinonasal symptoms in patients with olfactory symptoms and mild coronavirus disease2019 (COVID-19) and determining the patterns in emergence and resolution of olfactory/gustatory symptoms relative to general and sinonassal symptoms. This was a prospective cross-sectional study conducted at the outpatient otorhinolaryngology clinic at a COVID-19-designated referral Hospital. We included consecutive patients with new-onset olfactory dysfunction and positive polymerase chain reaction (PCR) assay of COVID-19. We asked the patients to fill in a questionnaire about general and sinonasal symptoms in association with anosmia, hyposmia or hypogeusia, and recorded the time course of the olfactory/gustatory symptoms during 2-weeks of follow-up. 76 patients with average age of $38.5 \pm 10.6$ years were included. Majority of participants $(94.7 \%)$ had general or sinonasal symptom. There was anosmia in $60.5 \%$ and hyposmia in $39.5 \%$, with sudden onset of olfactory symptoms reported in $63.2 \%$ of patients. During the follow-up, $30.3 \%$ of patients completely and $44.7 \%$ partially recovered from anosmia/hyposmia. Regardless of whether the general or olfactory symptoms appeared initially, the general symptoms resolved first while a degree of olfactory dysfunction persisted during the follow-up. Our study showed that hyposmia and anosmia in mild COVID-19 are frequently associated with general and sinonasal symptoms
\end{abstract}

Nasim Raad

nasim_raad@yahoo.com

1 Chronic Respiratory Disease Research Center, National Research Institute of Tuberculosis and Lung Diseases, Shahid Beheshti University of Medical Sciences, Masih Daneshvari Hospital, Neyavran, Darabad, Tehran, Iran and tend to persist longer than the general and sinonasal symptoms during the course of the disease.

Keywords COVID-19 · SARS-CoV-2 · Anosmia · Hyposmia · Hypogeusia

\section{Introduction}

Since January 2020 when the first cases of coronavirus disease-2019 (COVID-19) were reported from Wuhan, China [1], significant data on the pathobiology of severe acute respiratory syndrome-conoavirus-2 (SARS-CoV-2) has been accumulated at an accelerating speed in an attempt to keep pace with the nascent COVID-19 pandemic. Essential to the infectivity of the virus, the superficial spike protein of SARS-CoV-2 attaches to the angiotensin-converting enzyme 2 (ACE 2) receptor at the surface of the cells, particularly in the lungs [2], to gain access to and replicate inside the host cells. Thus, pneumonia is the dominant clinical feature of the hospitalized patients with the severe form of the disease. Clinical and imaging findings of the lower respiratory involvement including dyspnea [3], ground glass opacity and crazypaving appearance on computed tomography (CT) scan [4] are the main features of COVID-19; with respiratory complications including acute respiratory distress syndrome being the frequent cause of death $[5,6]$.

The expression levels of ACE2 receptors are different in various cell types in the upper airways [7, 8], and during the initial phase of pandemic, upper airway manifestations of COVID-19 were thought to be less common than the lower respiratory manifestations $[6,8]$. In an initial report from Wuhan, China, the frequency of olfactory symptoms was as low as $5.1 \%$ [9]. Since then, and in parallel with the 
global spread of COVID-19, much higher incidence of smell and taste disorders have been increasingly reported $[10,11]$. For instance, in a multicenter study performed in Europe, $85.6 \%$ of polymerase chain reaction (PCR) positive patients reported olfactory symptoms [12], prompting suggestions of a causal association between SARS-CoV-2 and smell and taste disorders [13].

Previous studies have investigated the prevalence of olfactory or gustatory symptoms in hospitalized patients or in patients with a spectrum of COVID-19 severity. In the present study, we have evaluated the general and sinonasal symptoms, particularly smell and taste disorders, in patients diagnosed with mild COVID-19 in the outpatient setting; focusing on the temporal patterns in the emergence and resolution of these symptoms in the course of COVID19 , and have explored the correlation of the smell and taste disorders with the general and sinonasal symptoms.

\section{Material and Methods}

\section{Patient Population and Study End Points}

This prospective single-centre cross-sectional study was performed at the outpatient department of a Hospital in Tehran, Iran. Since the beginning of the COVID-19 epidemic in our country, the Ministry of Health has designated this hospital as the referral center uniquely for admission of patients with COVID-19.

From March 1 to March 13, 2020, we prospectively collected data from consecutive adult patients ( $\geq 18$ years) presenting with new-onset anosmia who were clinically suspected as having mild COVID-19 but not fulfilling the criteria for hospital admission. The diagnosis of SARSCoV-2 infection was confirmed by real-time PCR performed on samples taken from the pharyngeal or nasopharyngeal swabs.

The following were the exclusion criteria: patients with olfactory or gustatory dysfunction before the COVID-19 pandemic and patients who were admitted to the hospital at the time of the study for any health issues including COVID-19. We asked the patients to fill in a questionnaire about their general symptoms including cough, myalgia, fever, headache and dyspnea and sinonasal including nasal obstruction, rhinorrhea, sneeze, nasal burn and facial pain. We divided the olfactory symptoms in three groups: anosmia for no sense of smell and hyposmia for reduced sense of smell and defined gustatory symptom (i.e., hypogeusia) as reduced sense of taste. We recorded the time course of the olfactory and gustatory symptoms at the initial visit and on a follow-up visit 2 weeks later. The study was approved by the ethical Committee of our hospital.

\section{Statistical Analysis}

Continuous variables are presented as means with standard deviation. The differences between the groups are examined using the Student's t test. Categorical data are compared with the $\chi^{2}$ or Fisher's exact test. The associations between the upper respiratory symptoms and the olfactory or gustatory findings are assessed using univariable logistic regression. Due to the relatively small number of participants, multivariable logistic regression was not performed to avoid overfitting the regression model. All statistical tests were 2-tailed. Statistical significance was set at a level of 0.05. Data were analyzed using SPSS Statistics Version 22.0 (IBM, New York, NY, USA).

\section{Results}

\section{Demographic and Clinical Characteristics of the Study Population}

The demographic and clinical characteristics including general and sinonasal symptoms are summarized in Table 1. A total of 76 patients with olfactory symptoms (anosmia or hyposmia) were included in the study. The average age was $38.5 \pm 10.6$ years (range 18-75) and 31 $(40.8 \%)$ were male.

Of the overall participants, 72 patients $(94.7 \%)$ had general or sinonasal symptoms. Four patients had isolated anosmia without general or sinonasal symptoms. The most common general symptoms in the study population consisted of cough in $36(47.4 \%)$, headache in $31(40.8 \%)$, dyspnea in 31 (40.8\%), myalgia in $23(30.3 \%)$ and fever in $9(11.8 \%)$ patients. The most common sinonasal symptoms in the overall participants were nasal obstruction in 19 $(25.0 \%)$ and rhinorrhea in $16(21.1 \%)$ patients.

There was no significant correlation between the general and sinonasal symptoms in the study population. Moreover, there was no significant correlation between the general symptoms (including headache) or sinonasal symptoms (nasal obstruction or rhinorrhea) and either olfactory or gustatory symptoms on logistic regression analysis (Table 2).

\section{Olfactory Manifestations of Patients with COVID- 19 in the Outpatient Setting}

A summary of the demographic and clinical findings in patients with olfactory manifestations are presented in Table 1 . Of the total participants, 46 patients $(60.5 \%)$ had anosmia and $30(39.5 \%)$ hyposmia. Olfactory symptoms had no significant differences between males and females. 
Table 1 Demographic and clinical characteristics

\begin{tabular}{|c|c|c|c|c|c|c|c|}
\hline $\begin{array}{l}\text { Total study participants } \\
(\mathrm{n}=76)\end{array}$ & $\begin{array}{l}\text { Olfactory } \\
\text { symptoms } \\
(\mathrm{n}=76)\end{array}$ & $\begin{array}{l}\text { Anosmia } \\
(\mathrm{n}=46)\end{array}$ & $\begin{array}{l}\text { Hyposmia } \\
(\mathrm{n}=30)\end{array}$ & $\begin{array}{l}p \text { value } \\
\text { (Anosmia vs. } \\
\text { Hyposmia) }\end{array}$ & $\begin{array}{l}\text { Gustatory } \\
\text { symptoms } \\
(\mathrm{n}=66)\end{array}$ & $\begin{array}{l}\text { No gustatory } \\
\text { symptoms } \\
(\mathrm{n}=10)\end{array}$ & $\begin{array}{l}p \text { value } \\
\text { (Gustatory vs. no } \\
\text { gustatory) }\end{array}$ \\
\hline Age & $38.5 \pm 10.6$ & $38.1 \pm 8.9$ & $39.1 \pm 1.8$ & 0.690 & $38.1 \pm 10.1$ & $41.7 \pm 13.7$ & 0.313 \\
\hline Male & $31(40.8 \%)$ & ${ }^{16}(51.6 \%)$ & 15 (48.4\%) & 0.235 & $24(77.4 \%)$ & $7(22.6 \%)$ & 0.080 \\
\hline Female & $45(59.2 \%)$ & $\begin{array}{l}30 \\
(66.7 \%)\end{array}$ & 15 & 0.09 & $42(93.3 \%)$ & $3(6.7 \%)$ & 0.053 \\
\hline \multicolumn{8}{|c|}{$\begin{array}{l}\text { Participants with general and } \\
\text { sinonasal symptoms } \\
(\mathrm{n}=72)\end{array}$} \\
\hline Fever & $9(11.8 \%)$ & $4(5.3 \%)$ & $5(6.6 \%)$ & 0.306 & $9(11.8 \%)$ & $0(0.0 \%)$ & 0.597 \\
\hline Myalgia & $23(30.3 \%)$ & ${ }^{12}(15.8 \%)$ & 11 & 0.326 & $20(26.3 \%)$ & $3(3.9 \%)$ & 0.984 \\
\hline Cough & $36(47.4 \%)$ & $\begin{array}{l}20 \\
(26.3 \%)\end{array}$ & 16 & 0.400 & $30(39.5 \%)$ & $6(7.8 \%)$ & 0.503 \\
\hline Dyspnea & $31(40.8 \%)$ & ${ }^{19}(25.0 \%)$ & ${ }^{12}(15.8 \%)$ & 0.910 & $27(35.5 \%)$ & $4(5.3 \%)$ & 0.957 \\
\hline Headache & $31(40.8 \%)$ & $19(25.0 \%)$ & $12(15.8 \%)$ & 0.910 & $28(36.8 \%)$ & $3(3.9 \%)$ & 0.514 \\
\hline Facial pain & $2(2.6 \%)$ & $2(2.6 \%)$ & $0(0.0 \%)$ & 0.516 & $2(2.6 \%)$ & $0(0.0 \%)$ & 0.577 \\
\hline Nasal obstruction & $19(25.0 \%)$ & 13 & $6(7.9 \%)$ & 0.416 & $18(23.7 \%)$ & $1(1.3 \%)$ & 0.438 \\
\hline Rhinorrhea & $16(21.1 \%)$ & $9(11.8 \%)$ & $7(9.2 \%)$ & 0.694 & $15(19.7 \%)$ & $1(1.3 \%)$ & 0.678 \\
\hline Sneeze & $6(7.9 \%)$ & $5(6.6 \%)$ & $1(1.3 \%)$ & 0.393 & $5(6.6 \%)$ & $1(1.3 \%)$ & 0.584 \\
\hline Nasal burning & $7(9.2 \%)$ & $5(6.6 \%)$ & $2(2.6 \%)$ & 0.697 & $7(9.2 \%)$ & $0(0.0 \%)$ & 0.584 \\
\hline
\end{tabular}

Table 2 Association of general and sinonasal symptoms with the olfactory or gustatory symptoms

\begin{tabular}{|c|c|c|c|c|}
\hline \multirow[t]{2}{*}{ General and sinonasal symptoms } & \multicolumn{2}{|c|}{ Anosmia versus hyposmia } & \multicolumn{2}{|c|}{ Gustatory versus no gustatory symptoms } \\
\hline & OR $(95 \% \mathrm{CI})$ & $p$ value & OR $(95 \% \mathrm{CI})$ & $p$ value \\
\hline Fever & $1.48(0.32-6.75)$ & 0.609 & 0.00 & 0.999 \\
\hline Myalgia & $1.50(0.46-4.8)$ & 0.491 & $0.97(0.17-5.4)$ & 0.978 \\
\hline Cough & $1.51(0.51-4.5)$ & 0.452 & $2.54(0.55-11.5)$ & 0.228 \\
\hline Dyspnea & $1.02(0.37-2.8)$ & 0.964 & $0.83(0.18-3.6)$ & 0.809 \\
\hline Headache & $0.77(0.23-2.6)$ & 0.685 & $0.61(0.1-3.5)$ & 0.586 \\
\hline Facial pain & $9.95(0.00)$ & 0.999 & 0.00 & 0.999 \\
\hline Nasal obstruction & $0.61(0.16-2.3)$ & 0.468 & $0.37(0.03-4.01)$ & 0.420 \\
\hline Rhinorrhea & $2.10(0.51-8.5)$ & 0.298 & $0.68(0.06-7.6)$ & 0.760 \\
\hline Sneeze & $0.23(0.02-2.5)$ & 0.233 & $1.44(0.12-17.1)$ & 0.769 \\
\hline Nasal burning & $1.00(0.15-6.6)$ & 0.999 & 0.00 & 0.999 \\
\hline
\end{tabular}

$C I$ confidence interval, $O R$ odds ratio

\section{Gustatory Manifestations of Patients with COVID- 19 in the Outpatient Setting}

A summary of the demographic and clinical findings in patients with gustatory manifestations are presented in
Table 1. Of the participants in the study, 66 patients $(86.8 \%)$ had a change in taste, all with hypogeusia. The mean age of patients with gustatory symptoms was $38.1 \pm 10.1$ years and was not different compared with patients with olfactory symptoms. 
There was no significant association between the gustatory symptoms and sex. Out of patients with hypogeusia, $42(63.6 \%)$ had concomitant anosmia and $24(36.4 \%)$ had hyposmia.

\section{Temporal Patterns in Development and Resolution of the Olfactory and Gustatory Symptoms and Their Relationship with General or Sinonasal Symptoms}

Of all the participants who developed general symptoms ( $\mathrm{n}=72), 41(56.9 \%)$ developed general symptoms first, 24 (33.3\%) developed olfactory symptoms first, and 7 (9.7\%) concomitantly developed general and olfactory symptoms. There was no difference in age or sex between the groups based on the order of development of general or olfactory symptoms, nor was there a difference in types of general or sinonasal symptoms (Table 3 ).

Among the 41 patients who developed general symptoms first, 30 patients $(73.2 \%)$ completely recovered from their general symptoms while a degree of the olfactory symptoms persisted at the end of the follow-up period. Of the 24 patients who developed olfactory symptoms first, 19 (79.1\%) completely recovered from their general symptoms while a degree of olfactory symptoms persisted at the end of the follow-up. There was a significant correlation between the relative onset of the general versus olfactory symptoms with the temporal course in improvement of these symptoms $(p=0.000)$.
During the 2-weeks follow-up period, 23 patients (30.3\%) completely recovered from anosmia or hyposmia, $34(44.7 \%)$ partially recovered, and there was no change in the olfactory symptoms in $19(25 \%)$ of patients (Table 4 ). There was no difference in the age or sex of patients who partially or completely recovered from the olfactory symptoms during the follow-up versus those who did not. Of the general or sinonasal symptoms, only facial pain was different between the recovery groups, with 2 patients $(10.5 \%)$ with facial pain having no recovery from anosmia during the follow-up ( $p=0.046)$ (Table 4).

The onset of the olfactory symptoms was sudden in 48 $(63.2 \%)$ and gradual in $28(36.8 \%)$ of patients. There was no statistically significant correlation between the sudden or gradual onset of the olfactory symptoms and recovery from the olfactory symptoms ( $p=0.510)$. Last, among the 42 patients with concomitant hypogeusia and anosmia, 27 patients $(64.3 \%)$ completely recovered from anosmia and gustatory symptoms within the follow-up period.

\section{Discussion}

Since the beginning of the severe acute respiratory syndrome-conoavirus-2 (SARS-CoV-2) epidemic in our country, there has been a sudden surge in the incidence of new cases with olfactory and gustatory disorders [10]. Reflecting the temporal and geographic distribution of the pandemic wave, subsequent studies from other countries in

Table 3 Patterns in development of the olfactory and general/sinonasal symptoms

\begin{tabular}{lllc}
\hline Characteristics & $\begin{array}{l}\text { Order of symptoms } \\
(\mathrm{N}=72 \text { with general/sinonasal and olfactory symptoms })\end{array}$ & $p$ value \\
\cline { 2 - 4 } & $\begin{array}{l}\text { General/sinonasal first } \\
(\mathrm{n}=41)\end{array}$ & $\begin{array}{l}\text { Olfactory first } \\
(\mathrm{n}=24)\end{array}$ & $\begin{array}{l}\text { Concomitant } \\
(\mathrm{n}=7)\end{array}$ \\
\hline Age & $39.1 \pm 9.5$ & $37.3 \pm 13.4$ & $39.8 \pm 7.5$ \\
Male & $14(48.3 \%)$ & $12(41.4 \%)$ & $3(10.3 \%)$ \\
Female & $27(57.1 \%)$ & $12(27.9 \%)$ & $4(9.3 \%)$ \\
General and sinonasal symptoms & & & 0.789 \\
Fever & $6(14.6 \%)$ & $2(8.3 \%)$ & 0.449 \\
Myalgia & $13(31.7 \%)$ & $9(37.5 \%)$ & $1(14.3 \%)$ \\
Cough & $23(56.1 \%)$ & $11(45.8 \%)$ & $2(14.3 \%)$ \\
Dyspnea & $17(41.5 \%)$ & $11(45.8 \%)$ & $3(28.6 \%)$ \\
Headache & $17(41.5 \%)$ & $11(45.8 \%)$ & $3(42.9 \%)$ \\
Facial pain & $2(2.8 \%)$ & $0(0.0 \%)$ & $3(42.9 \%)$ \\
Nasal obstruction & $11(26.8 \%)$ & $8(33.3 \%)$ & 0.751 \\
Rhinorrhea & $10(24.4 \%)$ & $4(16.7 \%)$ & 0.357 \\
Sneeze & $3(7.3 \%)$ & $2(8.3 \%)$ & 0.943 \\
Nasal burning & $4(9.8 \%)$ & $2(8.3 \%)$ & 0.943 \\
\hline
\end{tabular}


Table 4 Recovery from the olfactory symptoms during follow-up

\begin{tabular}{|c|c|c|c|c|}
\hline \multirow[t]{2}{*}{ Characteristics } & \multicolumn{3}{|c|}{ Recovery from the olfactory symptoms $(\mathrm{n}=76)$} & \multirow[t]{2}{*}{$p$ value } \\
\hline & $\begin{array}{l}\text { Complete } \\
(\mathrm{n}=23)\end{array}$ & $\begin{array}{l}\text { Partial } \\
(\mathrm{n}=34)\end{array}$ & $\begin{array}{l}\text { No recovery } \\
(\mathrm{n}=19)\end{array}$ & \\
\hline Age & $39.3 \pm 12.1$ & $37.8 \pm 10.5$ & $38.9 \pm 8.9$ & 0.852 \\
\hline Male & $7(22.6 \%)$ & $16(51.6 \%)$ & $8(25.8 \%)$ & 0.452 \\
\hline Female & $16(35.6 \%)$ & $18(40.0 \%)$ & $11(24.4 \%)$ & 0.521 \\
\hline \multicolumn{5}{|c|}{ General and sinonasal symptoms } \\
\hline Fever & $3(13.0 \%)$ & $4(11.1 \%)$ & $2(10.5 \%)$ & 0.969 \\
\hline Myalgia & $9(39.1 \%)$ & $8(23.5 \%)$ & $6(31.6 \%)$ & 0.449 \\
\hline Cough & $10(43.5 \%)$ & $17(50.0 \%)$ & $9(47.4 \%)$ & 0.890 \\
\hline Dyspnea & $10(43.5 \%)$ & $13(38.2 \%)$ & $8(42.1 \%)$ & 0.917 \\
\hline Headache & $8(34.8 \%)$ & $14(41.2 \%)$ & $9(47.4 \%)$ & 0.710 \\
\hline Facial pain & $0(0.0 \%)$ & $0(0.0 \%)$ & $2(10.5 \%)$ & 0.046 \\
\hline Nasal obstruction & $3(13.0 \%)$ & $8(23.5 \%)$ & $8(42.1 \%)$ & 0.093 \\
\hline Rhinorrhea & $6(26.1 \%)$ & $6(17.6 \%)$ & $4(21.1 \%)$ & 0.745 \\
\hline Sneeze & $3(13.0 \%)$ & $2(5.1 \%)$ & $1(5.3 \%)$ & 0.546 \\
\hline Nasal burning & $2(8.7 \%)$ & $3(8.8 \%)$ & $2(10.5 \%)$ & 0.974 \\
\hline
\end{tabular}

Europe have reported a similar increase in these symptoms associated with the COVID-19 outbreak [12, 14, 15]. While these studies examined the prevalence and association of the olfactory and gustatory symptoms with general or sinonasal symptoms of COVID-19, there is little data on the emergence and evolution of these symptoms in the course of COVID-19 [10].

In the present study, $\sim 33 \%$ of patients had anosmia as the initial symptom of COVID-19, which is similar to the rate of anosmia as the sole initial manifestation of COVID19 in hospitalized patients with a more severe form of the disease ( $\sim 39 \%$, our unpublished data). The difference in other manifestations of COVID-19 in the outpatient versus hospitalized patients may in part be due to a relatively higher viral load, with initial exposure causing anosmia, likely as a direct neuropathic effect rather than through a conductive mechanism, before systemic and lower respiratory symptoms are manifest, eventually leading to severe multi-organ involvement.

Although the frequent presence of the sinonasal symptoms simultaneously with the olfactory symptoms in patients with mild COVID-19 in the present study may point to predominant conductive rather than sensory neural mechanism for olfactory loss, it is likely that many patients with isolated anosmia, especially at the beginning of the pandemic, were unaware of an association with COVID-19 and may have not sought medical attention-hence patients with concomitant anosmia and general or sinonsal symptoms are more represented in the current study. Moreover, the relative distribution of the olfactory symptoms in the outpatient setting in the present study versus hospitalized patients-i.e., a relatively higher prevalence of anosmia compared with hospitalized patients (60\% vs. 43\%) together with a relatively higher prevalence of sudden-onset anosmia (63\% versus 41\%) (our unpublished inpatient data)_-suggest that sensory-neural mechanism may also play a role in the olfactory disorder in the mild form of COVID-19. Furthermore, $63 \%$ of patients had hypogeusia associated with anosmia, while the rates of simultaneous gustatory and olfactory dysfunction was even higher $(75 \%)$ in hospitalized patients with COVID-19, thus pointing to a possible polyneuropathic involvement in both mild and severe forms of COVID-19.

Recovery of olfaction in the short period of 2-weeks in the present study may be secondary to resolution of nasal mucosal edema and inflammation or functional recovery of the olfactory sensory cells, which occurred independent of the acuity of olfactory loss. Complete recovery of the olfactory function occurred in $\sim 30 \%$ of patients within 2 weeks, which is seemingly lower than the early recovery rate $(44 \%)$ in a multicenter European study [12], but this difference is due to the fact that in the European study the recovery was only assessed in patients who recovered from COVID-19 and not in the entire study participants. A higher prevalence of anosmia in that study ( $80 \%$ vs. $60 \%$ in the present study) would implicate a lower recovery rate in the whole population since the initial degree of olfactory loss is one of the most important determinants of olfactory recovery-i.e., hyposmic patients are more than twice likely to recover to normal than anosmic patients $[16,17]$. 
Resolution of the general symptoms prior to complete recovery from the olfactory symptoms in the present study is consistent with other studies of COVID-19 [12] and may point to a longer time-course of post-viral olfactory loss as a potential sequela of COVID-19 akin to other viruses [18]. Therefore, longer-term studies are needed to establish the degree of recovery of olfaction in patients with anosmia of COVID-19. Additionally, whether persistence of the olfactory dysfunction despite resolution of the general and sinonasal symptoms has implications on continuous infectivity would be critical in the dynamics of COVID-19 transmission and needs further investigation.

Although the exact mechanisms through which SARSCoV-2 causes sensory-neural olfactory loss is unknown, but accumulating data support a peripheral or central neuropathic basis as the likely mechanisms. SARS-CoV-2, like SARS-CoV, infects the cells by fusion of its spike protein to the ACE2 receptor on target cells. This contact necessitates cleavage of the spike protein by the cell surface protease transmembrane protease serine 2 (TMPRSS2) [19]. Thus, in addition to the viral load, variation in expression of SARS-CoV-2 entry proteins in the respiratory epithelium may explain the difference of clinical presentation and severity of COVID- 19.

The nasal respiratory epithelium goblet, basal and ciliated cells highly express ACE2 and TMPRSS2 and may be the upper airway reservoir for the virus during the course of COVID-19 [7]. In addition, the olfactory epithelial support cells and stem cells, but not mature olfactory sensory neurons, express ACE2 and TMPRSS2. Infection of these support cells by SARS-CoV-2 could impair the olfactory function by direct or indirect mechanism, for instance by denuding the sensory epithelial cilia or failure to effectively renew the olfactory epithelium over time [8].

A potential route for the putative central nervous effects of SARS-CoV-2 is via specialized glia known as olfactory ensheathing cells that can supply axons with macromolecules by way of exosomes [20]. This pathway may also act as a route for ACE2-independent virus transfer to axons of the olfactory receptor neurons in the olfactory bulbs [20]. Nonetheless, we found no change in the olfactory bulb in a patient with isolated anosmia secondary to COVID-19 on magnetic resonance imaging in the subacute phase of the disease [21]. Further longitudinal imaging, including functional imaging, are needed to further elucidate the presence and peripheral versus central extent of the sensory-neural loss in COVID-19.

The present study has several limitations. This study was performed in a single center. Due to the risk of infectivity to staff, we did not perform direct smell identification tests and the data were gathered by a questionnaire. The smell identification tests would make grading and scaling of the olfactory impairment more objective.
In conclusion, hyposmia/anosmia associated with mildly symptomatic patients with COVID-19 is frequently associated with general and sinonasal symptoms and tends to persist longer than the general and sino-nasal symptoms. Several clinical features point to a probable mixed sensoryneural and conductive mechanism for olfactory symptoms in mild COVID-19. Further studies are needed to elucidate the pathobiologic basis for sensory-neural impact of SARSCoV-2 and the long-term course in evolution of the olfactory function in COVID-19.

\section{Funding None.}

Compliance with Ethical Standards

Conflict of interest The authors declare that they have no conflict of interest.

\section{References}

1. Zhou P, Yang XL, Wang XG, Hu B, Zhang L, Zhang W et al (2020) A pneumonia outbreak associated with a new coronavirus of probable bat origin. Nature 579:270-273

2. Li Y, Bai WZ, Hashikawa T (2020) Response to commentary on: "The neuroinvasive potential of SARS-CoV-2 may play a role in the respiratory failure of COVID-19 patients". J Med Virol 92:707-709

3. Fang Y, Zhang H, Xie J, Lin M, Ying L, Pang P et al (2020) Sensitivity of chest CT for COVID-19: comparison to RT-PCR. Radiology 296:E115-E117

4. Xu X, Yu C, Qu J, Zhang L, Jiang S, Huang D et al (2020) Imaging and clinical features of patients with 2019 novel coronavirus SARS-CoV-2. Eur J Nucl Med Mol I 47:1275-1280

5. Ruan Q, Yang K, Wang W, Jiang L, Song J (2020) Clinical predictors of mortality due to COVID-19 based on an analysis of data of 150 patients from Wuhan. China Intensive Care Med 46:846-848

6. Chen J, Qi T, Liu L, Ling Y, Qian Z, Li T et al (2020) Clinical progression of patients with COVID-19 in Shanghai, China. J Infect 80(5):e1-e6

7. Sungnak W, Huang N, Bécavin C, Berg M, Queen R, Litvinukova $\mathrm{M}$ et al (2020) SARS-CoV-2 Entry genes are most highly expressed in nasal goblet and ciliated cells within human airways. Nat Med 26:681-687

8. Brann D, Tsukahara T, Weinreb C, Logan DW, Datta SR (2020) Non-neural expression of SARS-CoV-2 entry genes in the olfactory epithelium suggests mechanisms underlying anosmia in COVID-19 patients. Biorxiv. https://doi.org/10.1101/2020-03-25009084

9. Mao L, Jin H, Wang M, Hu Y, Chen S, He Q et al (2020) Neurologic manifestations of hospitalized patients with coronavirus disease 2019 in Wuhan, China. JAMA Neurol 10(77):1-9

10. Bagheri SH, Asghari AM, Farhadi M, Shamshiri AR, Kabir A, Kamrava SK et al (2020) Coincidence of COVID-19 epidemic and olfactory dysfunction outbreak. Med J Islam Repub Iran 34:62. https://doi.org/10.1101/2020-03-23-20041889

11. Karimi-Galougahi M, Raad N, Mikaniki N (2020) Anosmia and the need for COVID-19 screening during the pandemic. Otolaryngol Head Neck Surg 163:96-97 
12 Lechien JR, Chiesa-Estomba CM, De Siati DR, Horoi M, Le Bon SD, Rodriguez A et al (2020) Olfactory and gustatory dysfunctions as a clinical presentation of mild-to-moderate forms of the coronavirus disease (COVID-19): a multicenter European study. Eur Arch Otorhinolaryngol 277:2251-2261

13. Kaye R, Chang D, Kazahaya K, Brereton J, Denneny JC (2020) COVID-19 anosmia reporting tool: initial findings. Otolaryngol Head Neck Surg 163:132-134

14. Menni C, Valdes A, Freidin M, Ganesh S, Moustafa JES, Visconti A et al (2020) Loss of smell and taste in combination with other symptoms is a strong predictor of COVID-19 infection. Medrxiv. https://doi.org/10.1101/2020.04.0520048421

15. Giacomelli A, Pezzati L, Conti F, Bernacchia D, Siano M, Oreni L et al (2020) Self-reported olfactory and taste disorders in SARS-CoV-2 patients: a cross-sectional study. Clin Infect Dis 71:889-890

16. Cavazzana A, Larsson $M$, Münch $M$, Hähner A, Hummel $T$ (2018) Postinfectious olfactory loss: a retrospective study on 791 patients. Laryngoscope 128:10-15
17. London B, Nabet B, Fisher AR, White B, Sammel MD, Doty RL (2008) Predictors of prognosis in patients with olfactory disturbance. Ann Neurol 63:159-166

18. Seiden AM (2004) Postviral olfactory loss. Otolaryngol Clin North Am 37:1159-1166

19. Hoffmann M, Kleine-Weber H, Schroeder S, Krüger N, Herrler T, Erichsen S et al (2020) SARS-CoV-2 cell entry depends on ACE2 and TMPRSS2 and is blocked by a clinically proven protease inhibitor. Cell 181:271-280.e8

20. Butowt R, Bilinska K (2020) SARS-CoV-2: olfaction, brain infection, and the urgent need for clinical samples allowing earlier virus detection. ACS Chem Neurosci 11:1200-1203

21 Karimi-Galougahi M, Ghorbani J, Bakhshayeshkaram M, SafaviNaeini A, Haseli S (2020) Olfactory bulb magnetic resonance imaging in SARS-CoV-2-induced anosmia: the first report. Acad Radiol 27:892-893

Publisher's Note Springer Nature remains neutral with regard to jurisdictional claims in published maps and institutional affiliations. 